\title{
Transition to ultrasound as the first-line imaging modality for midgut volvulus: keys to a successful roll-out
}

\author{
HaiThuy N. Nguyen ${ }^{1,2}$ (1) Marla B. K. Sammer ${ }^{1,2} \cdot$ Matthew G. Ditzler $^{1,2} \cdot$ Lynn S. Carlson $^{1,2} \cdot$ Ray J. Somcio ${ }^{1,2}$. \\ Robert C. Orth ${ }^{3}$. J. Ruben Rodriguez ${ }^{4,5}$. Victor J. Seghers ${ }^{1,2}$
}

Received: 24 May 2020 / Revised: 23 September 2020 / Accepted: 9 November 2020 / Published online: 8 January 2021

(C) Springer-Verlag GmbH Germany, part of Springer Nature 2021

\section{Introduction}

In children, midgut volvulus has a reported bowel necrosis frequency of 9.3-17.6\% and mortality of 5.6-15.3\% [1-3]. The imaging exam typically used to diagnose midgut volvulus is a fluoroscopic upper gastrointestinal (GI) series. This study requires the physical presence of a radiologist [4], and for studies performed at remote locations or after-hours (e.g., evenings, weekends), either a radiologist needs to be called in from home or the child transferred to a hospital where the procedure is available. This process can lead to a delay in diagnosis and, most important, it can delay emergent surgical treatment and negatively impact patient outcomes.

Although upper GI series has a reported sensitivity of 93$100 \%$ for midgut malrotation $[2,5-8]$, its sensitivity for midgut volvulus is lower, ranging 54-89\% [7-10]. The published sensitivity and specificity of US for midgut volvulus are 83$96 \%$ and $89-100 \%$, respectively [11-14]. US has several other advantages compared to upper GI series. First, US can be performed portably, bringing the imaging to the child when the child is unable or too unstable to travel. Additionally, while upper GI series requires the oral ingestion or enteric

HaiThuy N. Nguyen

hnnguyen@texaschildrens.org

1 Singleton Department of Radiology, Texas Children's Hospital, 6701 Fannin St., Houston, TX 77030, USA

2 Department of Radiology, Baylor College of Medicine, Houston, TX, USA

3 Department of Pediatric Radiology, Children's Hospital of Wisconsin, Milwaukee, WI, USA

4 Department of Pediatric Surgery, Texas Children's Hospital, Houston, TX, USA

5 Department of Surgery, Baylor College of Medicine, Houston, TX, USA tube administration of contrast agent, no contrast agent is needed for US, although administration of fluid might be an aid to US for determining duodenal course $[11,13,15,16]$. Furthermore, US is free of ionizing radiation in comparison to upper GI series, with a reported average effective dose in infants ranging $1.6-3.2 \mathrm{mSv}[17,18]$. Finally, US is especially advantageous relative to upper GI series in settings where sonographers are on site around the clock.

At our institution, we developed and implemented a protocol to use US as the first-line imaging modality in pediatric patients with symptoms concerning for midgut volvulus. This was instituted to improve patient care by speeding time to diagnosis and surgical treatment given our large metropolitan area, where we cover emergent studies remotely at multiple hospitals without an on-site radiologist. By sharing our experience, we hope to provide a template for other institutions that elect to transition to US as a first-line imaging modality for diagnosing midgut volvulus. This would be useful in most settings but might be most helpful in geographically remote locations, high-volume practices, disaster scenarios where onsite radiologists are limited, and centers with a limited pediatric radiologist call pool. In reviewing our transition to using US as a first-line imaging modality, we outline what we believe are keys to successfully transitioning, including education, clinician buy-in and quality assurance (Table 1).

\section{Background}

\section{Local environment}

Our institution is a quaternary-care pediatric academic health system based in a large metropolitan area in North America and includes a primary pediatric teaching hospital, an adult hospital that focuses on women, mothers and babies, two community pediatric hospitals and seven outpatient pediatric specialty care centers. There are 994 licensed beds (834 at the 
Table 1 Summary of the three key steps and corresponding tips for a successful roll-out of focused abdominal US as a firstline modality for midgut malrotation and volvulus

\begin{tabular}{|c|c|}
\hline Steps & Tips \\
\hline \multicolumn{2}{|l|}{ Education } \\
\hline Sonographers & $\begin{array}{l}\text { - Publish imaging protocol } \\
\text { - Add volvulus to pylorus and intussusception exams } \\
\text { - Include volvulus protocol on competency checklist } \\
\text { - Provide hands-on scanning sessions with live mock patient } \\
\text { - Shadow sonographer and radiologist champions }\end{array}$ \\
\hline Radiologists and trainees & $\begin{array}{l}\text { - Create standardized report for checklist and consistency } \\
\text { - Share cases via secure group chats and case conferences } \\
\text { - Require self-directed on-line training module } \\
\text { - Provide hands-on scanning sessions with live mock patient }\end{array}$ \\
\hline \multicolumn{2}{|l|}{ Clinician buy-in } \\
\hline $\begin{array}{l}\text { Obtaining champions from key } \\
\text { specialties }\end{array}$ & $\begin{array}{l}\text { - Conduct face-to-face meetings with champions from the } \\
\text { emergency, surgery and neonatology departments } \\
\text { - Discuss positive US exams in real time, one-on-one } \\
\text { - Show example cases in multidisciplinary conferences }\end{array}$ \\
\hline $\begin{array}{l}\text { Ensuring that the upper GI series is still } \\
\text { performed in a timely manner }\end{array}$ & $\begin{array}{l}\text { - Create order set to pair US with upper GI series to ensure the } \\
\text { on-call radiologist is activated to perform the upper GI series, } \\
\text { though upper GI might be cancelled pending US results }\end{array}$ \\
\hline \multicolumn{2}{|l|}{ Quality assurance } \\
\hline $\begin{array}{l}\text { Obtaining preliminary data to } \\
\text { support US }\end{array}$ & $\begin{array}{l}\text { - Conduct literature review and synopsis } \\
\text { - Perform pilot study for interrater reliability and feasibility with } \\
\text { multiple sonographers }\end{array}$ \\
\hline $\begin{array}{l}\text { Tracking turnaround times and ordered } \\
\text { exam types }\end{array}$ & $\begin{array}{l}\text { - Identifies outliers in use of upper GI series } \\
\text { - Ensures diagnosis is not slowed by using US }\end{array}$ \\
\hline Keeping track of false negatives & $\begin{array}{l}\text { - Automatically generated monthly surgical cases of midgut } \\
\text { malrotation and volvulus from the EMR allow identification of } \\
\text { potential false negatives } \\
\text { - Separate radiology procedure code for the volvulus US allows } \\
\text { for auto population of the standardized reporting template and } \\
\text { database tracking }\end{array}$ \\
\hline
\end{tabular}

EMR electronic medical record, GI gastrointestinal, US ultrasound

${ }^{\text {a }}$ Our institutional imaging protocol for midgut volvulus US appears in Table 2 primary teaching hospital, and 86 and 74 beds at each of the community hospitals). Both community pediatric hospitals are full-service hospitals, including inpatient and outpatient services, a pediatric intensive care unit (PICU), an emergency center and operating suites. At one of the community hospitals, there is also a level III neonatal intensive care unit (NICU). The two community hospitals are located 23 miles and 38 miles from the primary teaching hospital, and the two community hospitals are 45 miles apart from each other.

The radiology departments at all of the hospitals offer US, CT, MRI, fluoroscopy, interventional radiology and radiography. In-house sonographers and CT and radiography technologists are available $24 \mathrm{~h}$ a day, 7 days a week (24/7). Five specialty-care centers offer US during the day, including same-day add-on exams that might need urgent surgery such as for appendicitis and hypertrophic pyloric stenosis.
However, because of the lack of fluoroscopy and operating room suites at specialty-care and urgent-care centers, evaluation for conditions requiring emergent surgery, such as midgut volvulus, is not performed at those locations.

Throughout the pediatric health system, there are 28 LOGIQ E9 and E10 machines (GE Healthcare, Chicago, IL). We have 34 pediatric sonographers and 21 body radiologists, all of whom are capable of performing and interpreting focused abdominal US for midgut volvulus. The average and median years of experience of our pediatric sonographers are 12.9 years and 12.5 years, respectively (range $1-32$ years). The average and median years of experience of our pediatric radiologists are 11.6 years and 9.5 years, respectively (range 1-34 years). In 2019, 1,276 focused abdominal US examinations were performed (i.e. volvulus, pylorus and necrotizing enterocolitis exams). 


\section{Impetus for change}

At least one body radiology attending physician is on site at each of the three hospitals during weekday hours (Monday through Friday, 8 am to $5 \mathrm{pm}$ ). A body radiologist attending physician and trainee are on-site at our primary teaching hospital 24/7, enabling all imaging exams to be interpreted by an attending radiologist shortly after being performed. Body radiologists are on call after-hours and on weekends at the community hospitals for emergent fluoroscopic procedures such as upper GI series. When activated, the community call radiologist must be on-site within $2 \mathrm{~h}$. An in-depth description of our community radiology operations has been described [19]. For emergent and urgent US performed remotely, sonographers discuss their images in person or over the phone with the radiologist at the primary teaching hospital before completing their exam. This allows the radiologist to support the sonographer and request additional imaging if needed.

As part of our process of continual quality improvement for community operations, we frequently consider opportunities to reduce the turnaround from imaging order placement to exam completion to try to improve patient satisfaction and outcomes. These efforts are aimed at improving disposition and discharge times for emergency center and inpatients and reducing the length of time to definitive treatment. Performance of emergent after-hours fluoroscopic studies is viewed as a necessary support for the medical and surgical specialties to allow their growth within the community hospitals. Transferring patients from community hospitals to the main teaching hospital is not desired because the core mission of the community hospitals is to improve and extend pediatric clinical care in the metropolitan region, allowing high-quality and safe clinical care to come to the patient. However, with off-site radiologist support and an activation window of up to $2 \mathrm{~h}$, after-hours community fluoroscopic procedures require relatively longer wait times compared to other imaging modalities that can be performed with an on-site technologist, such as US. Because US for detection of intestinal malrotation complicated by midgut volvulus has been reported to have superior sensitivity and specificity, we explored the feasibility of using US as a first-line imaging modality for midgut volvulus.

\section{Implementation}

\section{Process}

Sonographic findings of malrotation and midgut volvulus have historically been diagnosed across a variety of our US exams, including US of the complete abdomen, pylorus and intussusception studies. To improve our ability to track and improve quality of image acquisition and interpretation, we created a new imaging procedure code (IMG) called "US volvulus." We paired the standalone US volvulus IMG code with the upper GI series IMG code to create a combination order set for imaging workup of malrotation and midgut volvulus (Fig. 1). This ensures that US volvulus is included in the imaging workup and allows a single order to activate scheduling and operational workflows for both US and upper GI series exams, addressing concerns that a false-negative or inconclusive US could cause delay in obtaining an upper GI series.

In the community, use of the combination order ensures that the fluoroscopy staff members are aware of the case and the on-call radiologist is activated to drive in before the US volvulus is even performed. At the community sites, the US volvulus is performed by the on-site sonographer and reported by the radiologist working at the primary teaching hospital. If the exam is positive for midgut volvulus (i.e. whirlpool sign with or without dilatation of the proximal duodenum), and pending discussion with the surgeon, the patient can go directly to surgery without an upper GI series (Fig. 1). In that scenario the upper GI series is cancelled and the community oncall radiologist is contacted en route and informed of the clinical decisions. At the primary teaching hospital, where a pediatric radiologist is on site $24 / 7$, the same protocol is also followed, with a US volvulus performed first for suspected midgut volvulus and the ordered upper GI series completed based on the US findings. If the US volvulus is inconclusive or if the surgeon requests additional diagnostic confirmation with upper GI series prior to taking the patient to surgery, an upper GI series is performed without undue delay.

\section{Education of sonographers, radiologists and trainees}

We identified three distinct groups as targets for education: sonographers, radiologists and trainees (residents and fellows). Prior to launching the new malrotation and midgut volvulus imaging algorithm, we sought input from leaders in both the US and education sections. Education was both formal and informal and evolved based on feedback from targeted parties.

\section{Sonographers}

The new imaging algorithm (Fig. 1) and US volvulus protocol (Table 2) were uploaded onto the sonographer team website to increase understanding and proper utilization. The radiologist project leaders offered noon conferences detailing the embryology, pathology, algorithm and protocol for malrotation and midgut volvulus, enabling bidirectional feedback and improvement. Sonographers were asked to shadow pediatric sonographer and radiologist "champions" who had experience with using US to identify the superior mesenteric vasculature and the proximal duodenum, two main components of the US volvulus exam. To enhance the sonographers' skillset for 
a. More likely in infants

$<1$ year old. Bilious or

nonbilious emesis,

abdominal distention,

abdominal pain

b. Portable versus in

radiology based on

clinical status and

stability. If portable,

indicate AP and lateral

decubitus/cross table lateral

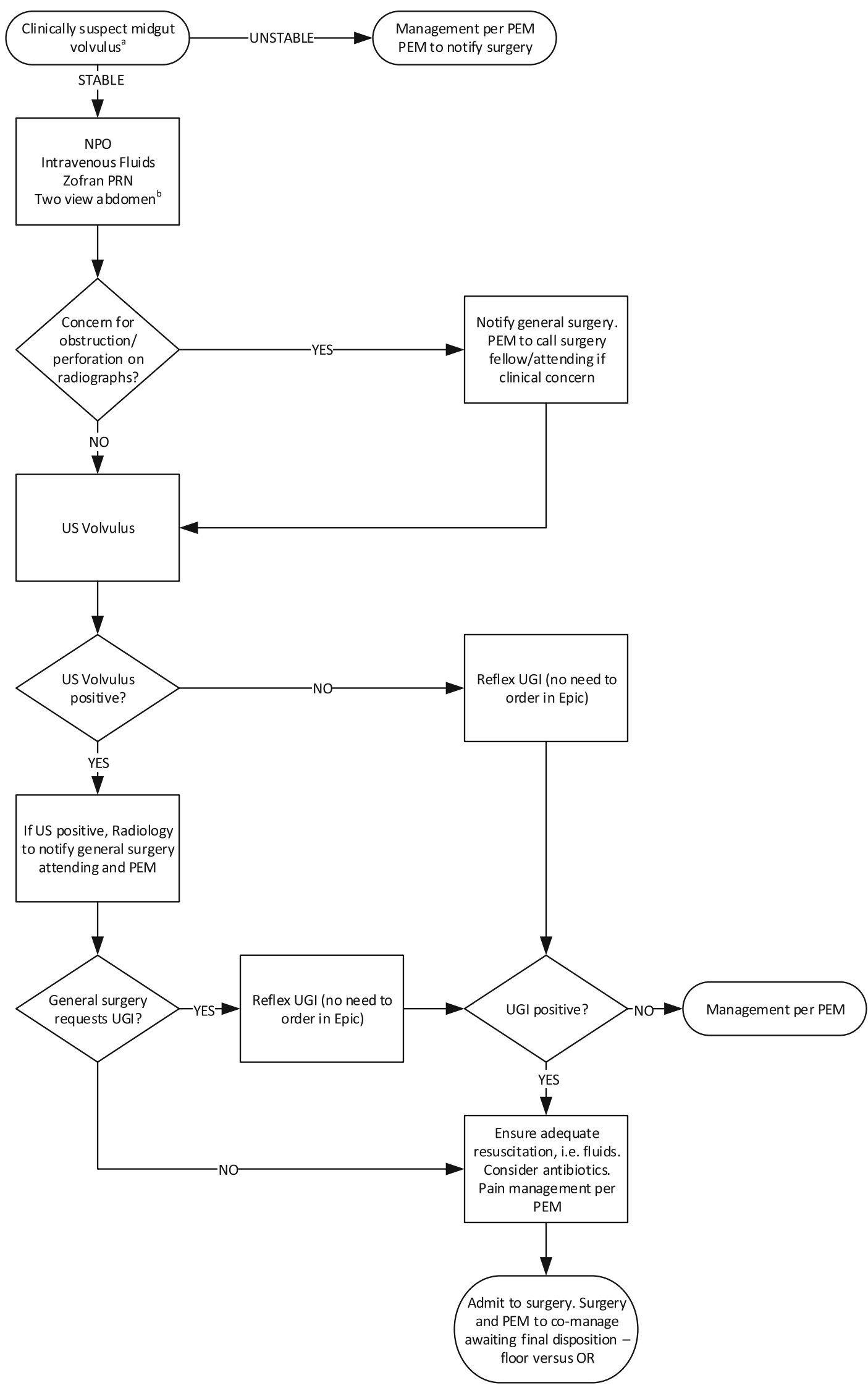


Fig. 1 Malrotation and midgut volvulus algorithm at Texas Children's Hospital starting in 2018. Of note, at the request of the General Surgery department, upper gastrointestinal series can still be performed in the presence of a positive US. AP anteroposterior, $N P O$ nil per os, $O R$ operating room, $P E M$ pediatric emergency medicine, $P R N$ pro re nata (when necessary), UGI upper gastrointestinal series

evaluating these components, and because volvulus has historically been identified on other abdominal US examinations, we added these components to other commonly ordered focused abdominal US protocols (US pylorus and US intussusception). Finally, we made US volvulus one of the exams requiring sonographer competency check-off prior to the sonographer being able to perform the exam.

\section{Radiologists and trainees}

We conducted a pilot study to assess the baseline ability of radiologists to diagnose malrotation and midgut volvulus on US and also to understand what needed to be emphasized in training radiologists. Three radiologists $(0-10$ years of experience post pediatric radiology fellowship) performed a blinded independent review of 31 US examinations (13 cases malrotation and midgut volvulus, 1 malrotation without midgut volvulus, and 17 normal). Prior to the blinded review, the three radiologists were given a self-directed PowerPoint training module. In the module, four findings used on US to evaluate for malrotation and midgut volvulus were illustrated (whirlpool sign, superior mesenteric artery [SMA]/superior mesenteric vein [SMV] relationship, duodenal dilation and duodenal location), as well as how these findings can be used to arrive at the impression. We determined interobserver agreement for each of the findings and the impression, and we calculated sensitivity and specificity for midgut volvulus.

In the pilot study, there was excellent agreement for the diagnosis of midgut volvulus among the three radiologists (intraclass correlation coefficient [ICC] 0.90, 0.83-0.95), as well as high sensitivity and specificity of US (100\% and 95$100 \%$, respectively). For three of the four findings, agreement was excellent among the readers (Table 3) [20]. For the fourth, there was good agreement among the readers regarding the location of the third portion of the duodenum. The US exams that were included had been performed by 17 sonographers. This pilot study suggested that (1) we could obtain highquality diagnostic exams with a heterogeneous sonographer group and (2) radiologists could consistently interpret the exams with minimal training.

Following this pilot study, we made the teaching module available to all 21 body radiologists for education and reference and updated it to emphasize the normal duodenal (especially 3 rd portion) anatomy. The module was also added to the trainees' core curriculum. We also provide trainees, sonographers and interested radiologists with intermittent opportunities for live demonstration of sonographic technique and normal bowel rotation on volunteer infants. We discuss the self-directed didactic material during these hands-on sessions.

To both educate and ensure that relevant imaging components of the US volvulus exam are properly interpreted, we created a standardized imaging report template (Fig. 2). This template auto-populates in our voice-recognition dictation program (Powerscribe 360; Nuance Communications, Burlington, MA) upon opening the exam in the picture archiving and communication system (PACS; Philips Intellispace, Foster City, CA). It provides the radiologists with a checklist of findings and how the findings support the standardized impression. The benefit of a standardized impression is that it conveys uniformity among all 21 body radiologists and might improve the degree of confidence with which radiologists convey the findings and impression in the report.

Positive cases, challenging cases, and cases where normal midgut anatomy is well demonstrated are regularly shared with the radiologists and trainees via intradepartmental case conferences as well as informally via a listserv on our add-on
Table 2 Institutional imaging protocol for midgut volvulus ultrasound

\begin{tabular}{ll}
\hline Technique & Notes \\
\hline $\begin{array}{l}\text { Probe } \\
\text { Static images and cine clips }\end{array}$ & $15 \mathrm{~L}$ or $9 \mathrm{~L}$; MSK setting preferred \\
$\begin{array}{l}\text { 1. Entire view of SMA/SMV with and without color in } \\
\text { dual screen }\end{array}$ & $\begin{array}{l}\text { - Must scan to the top of the bladder, even if there } \\
\text { is gas }\end{array}$ \\
& - Attempt to move gas by rolling patient \\
2. Pylorus and duodenal tip & - Look for "whirlpool" sign \\
3. Third portion of the duodenum & Look for duodenal dilation \\
4. Cecum with appendix & Should be located under the SMA and above the \\
& aorta \\
\hline
\end{tabular}

MSK musculoskeletal, SMA superior mesenteric artery, SMV superior mesenteric vein 
Table 3 Intraclass correlation coefficient (ICC) scores for the three readers

\begin{tabular}{llll}
\hline Question & ICC & $95 \%$ CI & ICC rating $^{\text {a }}$ \\
\hline Findings & & & \\
Is there a whirlpool sign? & 0.88 & $0.79-0.93$ & Excellent \\
Is the SMA/SMV normal? & 0.84 & $0.73-0.91$ & Excellent \\
Is the proximal duodenum dilated? & 0.83 & $0.72-0.90$ & Excellent \\
Is there a normal retroperitoneal third portion of the duodenum? & 0.73 & $0.57-0.84$ & Good \\
Impression & & & \\
Is there midgut volvulus? & $0.90^{\mathrm{b}}$ & $0.83-0.95$ & Excellent \\
\hline
\end{tabular}

$C I$ confidence interval, SMA superior mesenteric artery, $S M V$ superior mesenteric vein

${ }^{a}$ The Cicchetti guidelines for interpreting kappa or ICC inter-rater agreement measures were used and interpreted as follows: less than $0.40=$ poor, $0.40-0.59=$ fair, $0.60-0.74=$ good, $0.75-1.00=$ excellent [20]

${ }^{\mathrm{b}}$ Agreement and overall performance were best for the impression of midgut volvulus
PACS communicator (Prism; Nuance Communications, Burlington, MA). These discussions further educate the faculty, promote greater understanding of the imaging workflow algorithm, and created positive momentum for the transition toward US for the detection of midgut volvulus. Finally, rotating trainees, fellows and new faculty are asked to complete a local institutional online training educational module on the use of US in midgut volvulus.
Fig. 2 Standardized template for volvulus US in our voicerecognition software (we do not rely on US for diagnosing malrotation. However, it is included in our report template because identifying malrotation is integral to recognizing midgut volvulus. Per the institutional algorithm, upper gastrointestinal series is to follow US performed for bilious emesis, unless US is positive for midgut volvulus). D3 third portion of the duodenum, SMA superior mesenteric artery, $S M V$ superior mesenteric vein
TECHNIQUE: Grayscale ultrasonography of the bowel was performed in all 4 abdominal quadrants.

COMPARISON: [None].

FINDINGS:

Superior mesenteric vasculature:

- SMA/SMV alignment: [SMA/SMV relationship: Normal/Abnormal/Not visualized]

- Retroperitoneal D3 segment between aorta and SMA: [D3: Not assessable/Yes/No]

Cecum:

- [Cecal position: Normal/Not visualized/Abnormal]

Ascites/fluid collections: [None]

Other: [None]

IMPRESSION:

[Impression:1/2/3/4/5]

Alternative/additional diagnosis: [Alternative]

[Communication: Critical result/Significant findings]

1. No findings of midgut volvulus or malrotation = normal SMA and SMV relationship AND retromesenteric D3

2. No midgut volvulus = visualization of the entire superior mesenteric vascular pedicle without whirlpool sign; D3 is not visualized

3. Inconclusive for midgut volvulus = cannot see the retromesenteric D3 or entire superior mesenteric vascular pedicle

4. Findings of malrotation without midgut volvulus = intraperitoneal D3 without whirlpool sign AND abnormal SMA/SMV relationship or abnormal position of cecum

5. Malrotation with midgut volvulus = clockwise whirlpool sign $+/$ - dilated proximal duodenum 


\section{Clinician buy-in}

To implement US as the first-line imaging modality for midgut volvulus, we engaged key leaders from emergency medicine, surgery and neonatology. Data from the literature and imaging findings of midgut volvulus on US were presented during weekly surgery-radiology-pathology conferences. Champions from the key departments at all three pediatric hospitals assisted in creating a malrotation and midgut volvulus algorithm (Fig. 1). The newly created combined US and upper GI series order set ensured that the upper GI series would not be significantly delayed by performing US. Mitigating potential delays in patient care was vital to achieving the support of referring providers. Additionally, community clinical leaders working at sites without on-site radiologists after-hours or on weekends viewed positively the opportunity to improve the time from imaging order to diagnosis and surgical disposition. The algorithm was implemented with support of all stakeholders in January 2018.

From our surgical colleagues' perspective, a large radiology group across three hospitals with varying experience and diverse backgrounds with sonography contributed to hesitancy to evolve from established practices. One-on-one discussions to review key imaging findings on US at critical decision-making points in the management of these patients were crucial to overcoming surgeon biases against switching imaging modalities. This is analogous to when the surgeon is in the fluoroscopic suite and watching the upper GI series in person and making the decision to take the patient to surgery in real time. Through this combination of leadership involvement, multidisciplinary conferences, and one-on-one discussions, awareness of the new algorithm and confidence in the diagnostic accuracy of US exams increased. As discussed later, a growing number of cases with positive US for volvulus for which the upper GI series was cancelled prior to surgery indicates that the new algorithm has been well adopted.

\section{Quality assurance}

Since the new algorithm was implemented in January 2018, we have assessed metrics quarterly to ensure that quality of patient care has been maintained. To ensure all children diagnosed with midgut volvulus are included in ongoing analysis, we created recurring reports from both the electronic medical record (EMR; EPIC, Madison, WI) and radiology information system (RIS; Prism, Burlington, MA). The EMR report lists all children treated surgically for malrotation or midgut volvulus based on diagnosis codes. The RIS report lists all upper GI series and US exams performed for volvulus, pyloric stenosis, acute neonatal abdomen and intussusception. Data regarding cases - including outcomes, types of imaging and diagnosis on imaging, and time-point metrics - are collected and stored using REDCap electronic data capture tools hosted at Texas Children's Hospital [21].

From January 2018 through April 2020 we treated 16 children for midgut volvulus who were imaged using the new protocol. Although it is premature to report definitive data analysis, we have seen some trends. Despite growth in overall community imaging volume, use of after-hours upper GI series for midgut volvulus has not similarly increased and has actually trended down, while use of only US for diagnosing volvulus has increased (Fig. 3). We have also noted variability across the campuses (Table 4). For example, among the two community hospitals, one performed more exams overall,

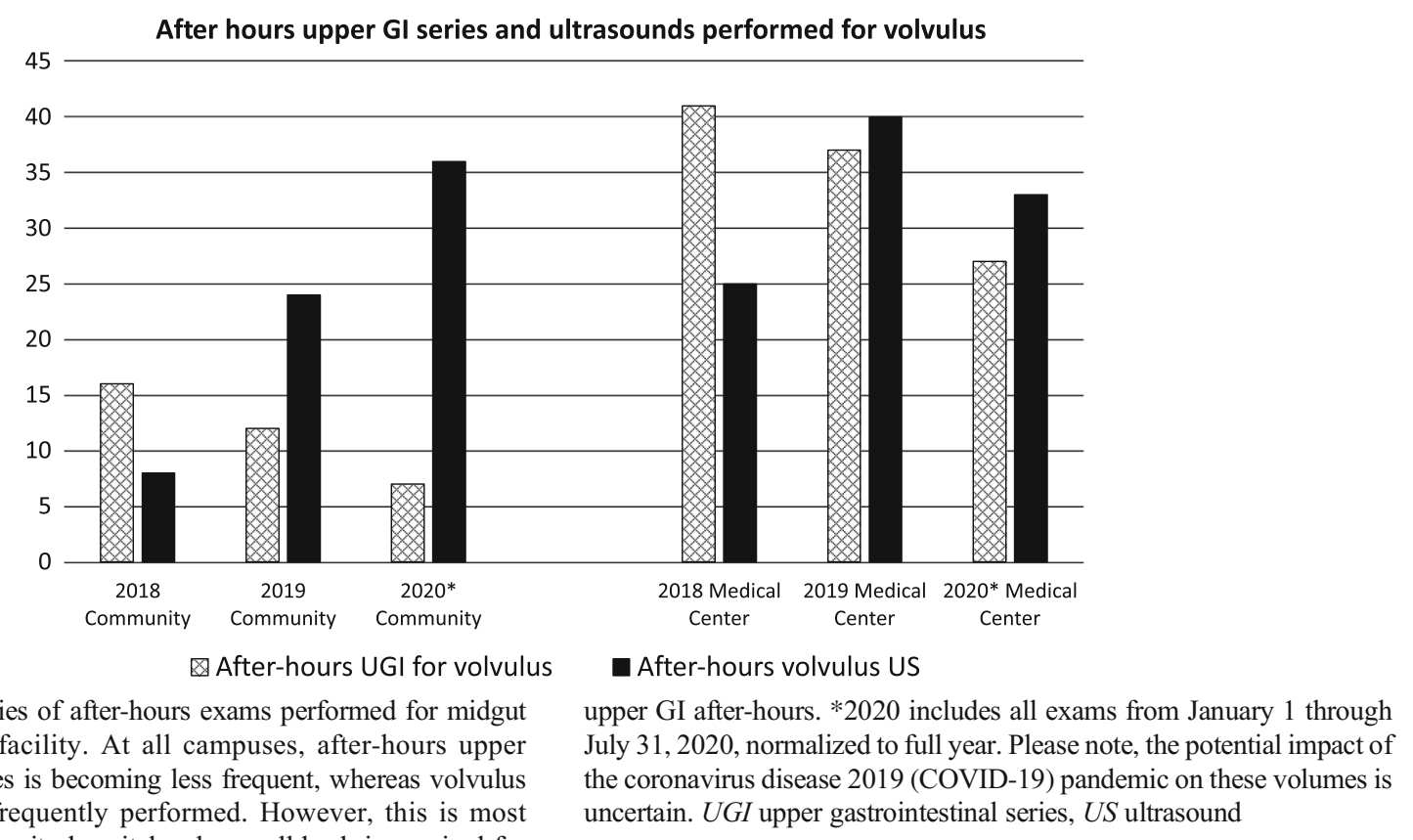


Table 4 Types of exams performed on children with surgically corrected midgut volvulus imaged between January 2018 and April 2020 using the new imaging algorithm

\begin{tabular}{lllll}
\hline Location & Number & US only & US + upper GI series & $\%$ US only \\
\hline Community & 11 & 5 & 6 & $45 \%$ \\
$\mathrm{CH}^{\mathrm{a}}$ & 7 & 4 & 3 & $57 \%$ \\
$\mathrm{CH}^{\mathrm{a}}$ & 4 & 1 & 3 & $25 \%$ \\
Medical Center & 5 & 3 & 2 & $60 \%$ \\
Total & 16 & 8 & 8 & $50 \%$ \\
\hline
\end{tabular}

GI gastrointestinal

${ }^{a} \mathrm{CH} 1$ and $\mathrm{CH} 2$ represent our two community pediatric hospitals and are subsets of "Community." The relatively low numbers limit definitive conclusions but they suggest that $\mathrm{CH} 2$ is a location where additional efforts might be focused. Of note, $\mathrm{CH} 2$ lacks a neonatal intensive care unit (NICU) and is staffed by a heterogeneous group of physicians who rotate from the main teaching hospital, which might account for fewer cases overall and the lowest percentage of US-only diagnoses determining surgical disposition. In distinction, $\mathrm{CH} 1$ has a NICU and uses a dedicated physician staffing model

with a greater incidence of using only US for diagnosis. This is attributed to the presence of a NICU affecting the number of patient transfers and a smaller dedicated physician staffing model, enabling easier communication and discussion between radiologists and surgeons and improved surgical acceptance for US.

The combination order set prospectively diagnosed midgut volvulus in 14 of 16 children (88\%). Of the other two, one was reported as high-grade proximal bowel obstruction and the other as malrotation with suspected intermittent volvulus. Overall, US prospectively reported malrotation with midgut volvulus in 13 of $16(81 \%)$. On the other three US exams, two reported proximal bowel obstruction and one was reported as nondiagnostic because of bowel gas. In retrospective review, the midgut volvulus could be identified on the US images in all three. On upper GI series, 3 of 8 (38\%) diagnosed midgut volvulus. The other five upper GI series were all reported as abnormal because of either proximal bowel obstruction or malrotation. Of note, in three of these five, volvulus had been reported on US, but the subsequent upper GI series was reported as high-grade proximal bowel obstruction, with deferral to US for the diagnosis of midgut volvulus.

During this time period, there was one false-positive exam in a child with a clockwise whirlpool sign, abnormal SMA/SMV relationship, and a large complex intraperitoneal fluid collection on US. An upper GI series subsequently demonstrated complete gastric outlet obstruction and gastric malposition. The duodenum was not assessed because of gastric outlet obstruction. At surgery, the child was diagnosed with segmental volvulus (rather than midgut volvulus) associated with a congenital mesenteric duplication cyst, which displaced the stomach and distorted the SMA/SMV. On retrospective review, a clue that the clockwise whirlpool sign was a segmental volvulus rather than midgut volvulus associated with congenital midgut malrotation was that the duodenum could not be identified in the whirlpool.

Turnaround times of all after-hours US and emergent upper GI series following the new imaging algorithm have been monitored. This is to confirm that the turnaround time for volvulus US is similar to that of other emergent focused abdominal US exams and that adding volvulus US to upper GI series for suspected volvulus did not substantially increase upper GI series turnaround times. Thus far, turnaround times on the combined protocols for volvulus patients have been faster than those of upper GI series alone, averaging $127 \mathrm{~min}$, compared with $154 \mathrm{~min}$ for after-hours upper GI series. As expected, US-alone turnaround times (average $68 \mathrm{~min}$ ) have remained faster than upper GI series times since the new protocol was implemented.

\section{Discussion}

The goal of our transition to US as the first-line imaging modality for the diagnosis of malrotation and midgut volvulus was to improve clinical care through increased diagnostic accuracy via a non-radiation-emitting examination and to decrease time from ordering an imaging exam to subsequent diagnosis and surgical disposition. Malrotation and midgut volvulus are relatively rare entities, so establishing a new volvulus US IMG code was instrumental to organize and enable data tracking, which we also use to verify to our clinical partners the diagnostic success of US over time relative to upper GI series and surgical findings. Creating a combination imaging order set that includes both volvulus US and upper GI series ensured that US is included in the imaging workup but at no time-cost to the patient if an upper GI series is needed. The greatest barrier to use of US by the clinicians was the potential delay if an upper GI series was still deemed necessary, a concern exacerbated at our community hospitals where no on-site radiologists are available after-hours or on weekends. Simultaneously scheduling/ performing the volvulus US and activating the community call radiologist for the upper GI series was integral to addressing these concerns.

The heterogeneous makeup and size of our organization made it challenging to achieve institution-wide buyin from our surgical, emergency medicine and neonatology partners. With 21 pediatric radiologists and 18 pediatric surgeons throughout three pediatric hospitals, it did take some time before the surgeons were comfortable with making the decision to take a child to surgery solely on the basis of a positive US exam. Our successful transition 
benefitted from our decision to engage relevant clinical stakeholders in advance through discussion of the literature, demonstration of positive US findings that had been incidentally found by our own sonographers, and implementation of clear and concise standardized reports.

Instrumental to the successful transition was continued formal and informal education of the sonographers, trainees, radiologists and clinicians. Because volvulus is rare, multidisciplinary conferences where everyone can learn from the limited number of cases is vital. Additionally, key components of the volvulus US such as evaluating the SMA and SMV vascular pedicle and third portion of the duodenum should be routinely included in more common studies such as pylorus or intussusception US.

A secondary benefit of our new imaging algorithm for midgut volvulus is that community call activations for emergent after-hours upper GI series have trended down, despite overall increased community departmental imaging volume. The number of radiologists trained in pediatric radiology is progressively declining and call burden has been shown to contribute to burnout through high emotional exhaustion and depersonalization. Opportunities to shift practice from the upper GI series requiring on-site physician support to US utilizing remote physician support could help address the pediatric radiology shortage by improving the call burden for pediatric radiologists [22-24].

Implementing US as the first-line exam at institutions where after-hours US is the responsibility of trainees or radiologists who are not subspecialty-trained in pediatric radiology would be different from our experience. However, similar to a variety of other conditions (hypertrophic pyloric stenosis, ileocolic intussusception and appendicitis) that are now routinely diagnosed on US at both pediatric and adult facilities, US for the diagnosis of volvulus could be similarly incorporated into clinical practice, and not limited to pediatric institutions such as ours with $24 / 7$ pediatric specialty-trained attending coverage.

There are limitations to the analysis of our transition to US as a first-line imaging modality. Despite being a large pediatric health system, we see a low number of positive US exams for malrotation and midgut volvulus, reflective of their relatively low incidence in the population. This prevents definitive statistical analysis of US exams' diagnostic accuracy, and for this, we are pursuing a multi-institutional study. Additionally, given the small numbers, while our turnaround time metrics meet internal goals, the impact on patient outcomes is not known.

Finally, as described in quality assurance, in 3 of the 16 children surgically treated for midgut volvulus, the interpreting radiologist did not prospectively diagnose midgut volvulus on US. Two of these were reported as abnormal because of proximal small bowel obstruction, one nondiagnostic because of bowel gas. Additionally, we had one false positive, with a segmental volvulus overcalled a midgut volvulus. In retrospect, the duodenum was not clearly seen within the identified bowel volvulus. Based on these cases, we have changed our approach to both sonographer image acquisition and radiologist interpretation. First, we have reiterated the necessity of graded compression to decrease artifact from gas, particularly from a midline subxiphoid approach. Second, we now emphasize the importance of recognizing duodenal (proximal small bowel) dilation as a sign that should prompt further evaluation for midgut volvulus. To this end, we have included additional imaging of the proximal duodenum in the imaging protocol and also in the report. Additionally, we have added discussion in the training module. For further description and more examples, we collaborated with two other institutions to create a pictorial essay for US evaluation of midgut malrotation and volvulus [25]. Last, to reiterate the algorithm used at our institution, an upper GI series follows an inconclusive or nondiagnostic US. As partially indicated by the outcome of upper GI series in the 8 children with midgut volvulus who were imaged with upper GI series, only a minority ( 3 of 8 ) had the midgut volvulus identified on upper GI series. Consequently, we have found the combination of US followed by upper GI series especially useful in problem cases.

Despite the limitations, this manuscript is the first to detail key steps for systematically implementing US as a first-line imaging modality for malrotation and midgut volvulus. Given its adoption at both community and academic sites, our experience suggests that the use of US for primary diagnosis of midgut volvulus can be successful in a wide variety of settings. By describing our successful transition, we hope to aid institutions that might similarly benefit from this protocol.

\section{Conclusion}

An imaging protocol to use US as the first-line modality for diagnosing malrotation with midgut volvulus has been successfully deployed at our institution. This workflow required significant education and communication with many clinical partners. US can be implemented in a wide variety of settings, and this report can be used to guide others.

Acknowledgments The authors would like to thank Dr. Binita Patel, $\mathrm{MD}$, an associate professor at the Department of Pediatrics-Emergency Medicine at Texas Children's Hospital, Houston, TX, for her support and participation in creating and implementing the institution-wide midgut malrotation and volvulus algorithm.

\section{Compliance with ethical standards}

Conflicts of interest None 


\section{References}

1. Messineo A, MacMillan JH, Palder SB, Filler RM (1992) Clinical factors affecting mortality in children with malrotation of the intestine. J Pediatr Surg 27:1343-1345

2. Lin J, Lou C, Wang K (1995) Intestinal malrotation and midgut volvulus: a 15-year review. J Formos Med Assoc Taiwan Yi Zhi 94: $178-181$

3. Millar A, Rode H, Brown R, Cywes S (1987) The deadly vomit: malrotation and midgut volvulus. Pediatr Surg Int 2:172-176

4. Raske ME, Dempsey ME, Dillman JR et al (2015) ACR appropriateness criteria: vomiting in infants up to 3 months of age. J Am Coll Radiol 12:915-922

5. Torres A, Ziegler M (1993) Malrotation of the intestine. World J Surg 17:326-331

6. Applegate KE, Anderson JM, Klatte EC (2006) Intestinal malrotation in children: a problem-solving approach to the upper gastrointestinal series. Radiographics 26:1485-1500

7. Seashore J, Touloukian R (1994) Midgut volvulus: an ever-present threat. Arch Pediatr Adolesc Med 148:43

8. Sizemore AW, Rabbani KZ, Ladd A, Applegate KE (2008) Diagnostic performance of the upper gastrointestinal series in the evaluation of children with clinically suspected malrotation. Pediatr Radiol 38:518-528

9. Stephens LR, Donoghue V, Gillick J (2012) Radiological versus clinical evidence of malrotation, a tortuous tale - 10-year review. Eur J Pediatr Surg 22:238-242

10. Dilley AV, Pereira J, Shi ECP et al (2000) The radiologist says malrotation: does the surgeon operate? Pediatr Surg Int 16:45-49

11. Chao HC, Kong MS, Chen JY et al (2000) Sonographic features related to volvulus in neonatal intestinal malrotation. J Ultrasound Med 19:371-376

12. Zhou L, Li S, Wang W et al (2015) Usefulness of sonography in evaluating children suspected of malrotation: comparison with an upper gastrointestinal contrast study. J Ultrasound Med 34:18251832

13. Zhang W, Sun H, Luo F (2017) The efficiency of sonography in diagnosing volvulus in neonates with suspected intestinal malrotation. Medicine 96:e8287

14. Pracros J, Sann L, Genin G et al (1992) Ultrasound diagnosis of midgut volvulus: the "whirlpool" sign. Pediatr Radiol 22:18-20
15. Hennessey I, John R, Gent R, Goh DW (2014) Utility of sonographic assessment of the position of the third part of the duodenum using water instillation in intestinal malrotation: a single-center retrospective audit. Pediatr Radiol 44:387-391

16. Cohen HL, Haller JO, Mestel AL et al (1987) Neonatal duodenum: fluid-aided US examination. Radiology 164:805-809

17. Staton RJ, Williams JL, Arreola MM et al (2007) Organ and effective doses in infants undergoing upper gastrointestinal (UGI) fluoroscopic examination. Med Phys 34:703-710

18. Damilakis J, Stratakis J, Raissaki M et al (2006) Normalized dose data for upper gastrointestinal tract contrast studies performed to infants: normalized doses to infants from UGIC studies. Med Phys 33:1033-1040

19. Seghers VJ, Sher AC, Nguyen HN et al (2020) Development of pediatric academic-community radiology services: lessons learned. Pediatr Radiol 50:1207-1216

20. Cicchetti DV (1994) Guidelines, criteria, and rules of thumb for evaluating normed and standardized assessment instruments in psychology. Psychol Assess 6:284-290

21. Harris PA, Taylor R, Thielke R et al (2009) Research electronic data capture (REDCap) - a metadata-driven methodology and workflow process for providing translational research informatics support. J Biomed Inform 42:377-381

22. Ayyala RS, Ahmed FS, Ruzal-Shapiro C, Taylor GA (2019) Stressors contributing to burnout amongst pediatric radiologists: results from a survey of the Society for Pediatric Radiology. Pediatr Radiol 49:714-722

23. Farmakis SG, Hardy AK, Thomas KB et al (2019) Changes in factors influencing fellowship choices among radiology residents from 2008 to 2018 and methods that may increase interest in the pediatric radiology subspecialty. Pediatr Radiol 49:1132-1141

24. Pfeifer CM (2018) Declining interest in pediatric radiology prompts a call to action. J Am Coll Radiol 15:490-492

25. Nguyen HN, Navarro OM, Guillerman RP et al (2020) Untwisting the complexity of midgut malrotation and volvulus ultrasound. Pediatr Radiol. https://doi.org/10.1007/s00247-020-04876-x

Publisher's note Springer Nature remains neutral with regard to jurisdictional claims in published maps and institutional affiliations. 